\title{
Variable dust formation by the double-line spectroscopic binary WR 70 (HD 137603, WC9vd+BOI)
}

\author{
Peredur M. Williams \\ Institute for Astronomy, University of Edinburgh, \\ Royal Observatory, Blackford Hill, Edinburgh EH93HJ, Scotland, UK
}

Karel A. van der Hucht

SRON Nationaal Instituut voor Ruimteonderzoek, Sorbonnelaan 2, NL-3584CA Utrecht, Nederland

Fred Marang

South African Astronomical Observatory, P.O. Box 9, Observatory, 7935, South Africa

\begin{abstract}
Infrared photometry of the WC9vd+B0I spectroscopic binary WR 70 over two decades shows variable dust emission. The variations are relatively slow and show evidence for an underlying process with a period $P=1045 \pm 60 \mathrm{~d}$, together with modulation on shorter and longer time-scales.
\end{abstract}

Variability of WR 70 in the near-IR was reported by Williams, van der Hucht \& The (1987), based on comparison of their 1983- 86 observations from the SAAO and ESO, with earlier observations by Cohen (1975) and Pitault et al. (1983). We have continued IR-monitoring WR 70, to search for systematic variations. The synoptic light curves (Figure 1) show amplitude increasing with wavelength, consistent with the spectral energy distribution (SED) which showed dust emission, deviating from the stellar wind SED only longward of $1.5 \mu \mathrm{m}$ (Williams et al. 1987). The $J$-photometry suggests, that the stellar wind flux from WR 70 is constant to within a few per cent, and that variations at longer wavelengths must, therefore, be due to variable dust emission; which is present even at minimum. The light curves delineate clear features, including a sharp maximum in 1989.25 and a well defined fading and recovery in 1992-93.

The WC9 and B0I components of WR 70 display RV variations, showing it to be an SB2 (Golombek 1983; Niemela 1995) and, therefore, a collidingwind binary. This prompted a search for periods in the variation of the dust emission, which might be modulated by orbit-related effects. A CLEAN power spectrum (using PERIOD, Dhillon \& Privett 1997) of $128 \mathrm{~K}$-magnitudes gave a strong, broad maximum at $0.356 \mathrm{y}^{-1}(1026 \mathrm{~d})$. On the basis that the $(K-L)$ colour variations, attributable to dust formation starting and stopping, may be a better indicator of underlying processes occuring in a colliding-wind system, $126(K-L)$ colours were also searched, giving maximum power at $0.348 \mathrm{y}^{-1}$ $(1050 \mathrm{~d})$. The string-length method, useful when the form of the light curve is 


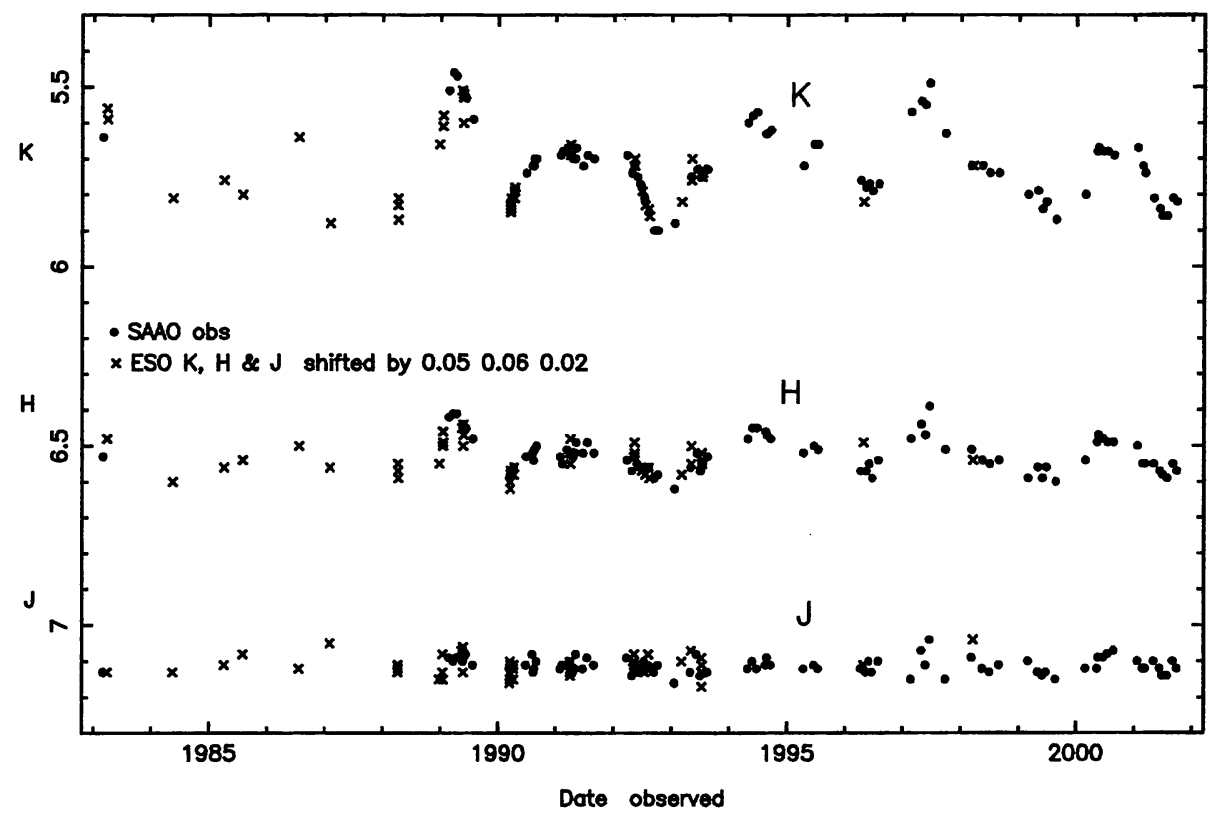

Figure 1. JHK light curves of WR 70 from observations at ESO ( $\times$; mostly $1983-93$ ) and SAAO (•; mostly $1989-2001$ ), combined by applying shifts determined from comparison of contemporaneous $(|\Delta T| \leq 6 \mathrm{~d})$ observations.

not known, gave a broad minimum at $0.36 \mathrm{y}^{-1}$ (1045 d) and clear minima at $2 \mathrm{P}$ and $3 \mathrm{P}$. These tests suggest that there is an underlying process in WR 70, modulating dust formation with a period of about $1045 \pm 50 \mathrm{~d}$, but light curves phased to this period show significant scatter, suggesting that other processes on both shorter and longer time-scales are at work, such as might be caused by high-density structures in one of the stellar winds, and a third stellar component in a longer period orbit, respectively.

\section{References}

Cohen, M. 1975, MNRAS 173, 489

Dhillon, V.S., Privett, G.J. 1997, PERIOD Time-Series Analysis Package, Starlink User Note 167.5, Rutherford Appleton Laboratory

Golombek, D.A. 1983, Estudio Espectrografico de Una Estrella Supergiante con Líneas de Emisión, $\mathrm{PhD}$ thesis, Universidad de Buenos Aires, Argentina

Niemela, V.S. 1995, in: K.A. van der Hucht \& P.M. Williams (eds), Wolf-Rayet Stars: Binaries, Colliding Winds, Evolution, Proc. IAU Symposium No.163 (Dordrecht: Kluwer), p. 223

Pitault, A., Epchtein, N., Gómez, A.E., Lortet, M.C. 1983 A\&A 120, 53

Williams, P.M., van der Hucht, K.A., Thé, P.S. 1987, A\&A 182, 91 